\title{
Cross-gain modulation in broad-area vertical-cavity semiconductor optical amplifier
}

\author{
Francesco Marino, ${ }^{\text {a) }}$ Luca Furfaro, and Salvador Balle \\ Institut Mediterrani d'Estudis Avançats, CSIC-UIB, E-07071, Palma de Mallorca, Spain
}

(Received 25 October 2004; accepted 3 March 2005; published online 8 April 2005)

\begin{abstract}
We demonstrate that broad-area vertical-cavity semiconductor optical amplifiers allow for wavelength conversion at $2.5 \mathrm{~Gb} / \mathrm{s}$ via cross-gain modulation (XGM). XGM is reached with a saturation beam of only $1.5 \mathrm{~mW}$ over an optical bandwidth of $0.7 \mathrm{~nm}(215 \mathrm{GHz})$. Depending on the wavelengths of the injected fields, inverted or noninverted output can be obtained. () 2005 American Institute of Physics. [DOI: 10.1063/1.1905811]
\end{abstract}

Semiconductor optical amplifiers (SOA) display nonlinear optical response on short time scales which arises from the changes induced by the injected optical field in both the total carrier density and its distribution over the energy bands. These ultrafast optical nonlinearities may allow for efficient all-optical signal processing; ${ }^{1}$ actually, all-optical wavelength conversion of the signal, data-format translation and add-drop functionalities have been demonstrated by using SOAs via cross-gain modulation ${ }^{2}$ (XGM), cross-phase modulation, ${ }^{3}$ or four-wave mixing. ${ }^{4}$ SOAs are usually of the traveling-wave type, which maximizes the optical bandwidth by strongly suppressing the ripples due to facet reflectivities. In order to suppress the sensitivity to polarization inherent to planar structures, specially when quantum-well active regions are used, SOAs require specific designs that make their coupling efficiency to optical fibers quite low.

Vertical-cavity SOAs (VCSOAs) have recently been recognized as an interesting alternative to SOAs. ${ }^{5,6}$ However, their single-pass gain is quite small, hence, they are necessarily of the Fabry-Pérot type with high-reflectivity mirrors. Thus, strongly wavelength-dependent amplification occurs only around the cavity resonances. The optical bandwidth can be increased by lowering the reflectivity of the mirrors at the cost of reducing the maximum gain. By optimizing this trade-off between gain and bandwidth, ${ }^{7}$ a gain of $11.3 \mathrm{~dB}$ and a corresponding optical bandwidth of $0.6 \mathrm{~nm}(100 \mathrm{GHz})$ were demonstrated in the $1.3 \mu \mathrm{m}$ wavelength range for an input signal power of $-20 \mathrm{~dB} \mathrm{~m} .^{8}$ For VCSOAs in the $980 \mathrm{~nm}$ wavelength range, a maximum gain of $20 \mathrm{~dB}$ and an optical bandwidth of $0.1 \mathrm{~nm}$ were measured for an input signal of $-40 \mathrm{~dB} \mathrm{~m}{ }^{9}$

The use of broad-area devices could provide a means to solve these limitations. ${ }^{14}$ In fact, the larger the device, the lower the frequency separation between the transverse modes and in broad-area VCSOAs (diameter of $50 \mu \mathrm{m}$ or more), the transverse resonances form a quasicontinuum under the gain curve. Due to its transverse dimension, broad area VCSOAs also provide a higher gain and wider optical band. In Ref. 14 we have demonstrated that a bottom-emitting, oxideconfined VCSOA with a diameter of $54 \mu \mathrm{m}$ that operates at wavelengths around $980 \mathrm{~nm}$ can reach a gain of $16.3 \mathrm{~dB}$ with $0.7 \mathrm{~nm}$ optical bandwidth for an optical input power of $0.1 \mathrm{~mW}$, the input saturation power being $1.4 \mathrm{~mW}$. The maximum gain was polarization independent, but the gain

\footnotetext{
${ }^{a)}$ Electronic mail: fmarino@imedea.uib.es
}

spectra for the two orthogonal polarization orientations were displaced by cavity birefringence. ${ }^{10}$

In this letter we explore the performances of broad-area VCSOAs as wavelength converters via XGM. We characterize the XGM efficiency as a function of the injected power, wavelength and polarization of the two beams. We show that XGM occurs for all wavelengths under the VCSOA gain spectrum. Finally, we estimate the modulation bandwidth of the process.

Our VCSOA is the one used in Ref. 14, with a circular oxide window of $54 \mu \mathrm{m}$ in diameter, Bragg mirrors consisting of $17 n$-type and $30 p$-type pairs, and operated in reflection mode. The device does not lase under $\mathrm{cw}$ operation, but its cw light-current curve corresponds to that of a lightemitting diode displaying thermal rollover for bias currents around $200 \mathrm{~mA}$. For a bias current of $185 \mathrm{~mA}$, the amplified spontaneous emission (ASE) spectrum is peaked around $\lambda$ $=982.80 \mathrm{~nm}$, and the total ASE power is $\sim 1.4 \mathrm{~mW}$. By measuring the ASE power after passing through the collimator, we estimate the coupling losses to be around $0.3 \mathrm{~dB}$.

We then inject two monochromatic beams in the VCSOA cavity which overlap in space and both enter into the VCSOA along the optical axis. The injection beams are provided by two tunable external cavity lasers, isolated from the VCSOA by optical diodes. The two injection beams can be independently controlled in wavelength, power, and polarization. The wavelengths are measured by a monochromator (resolution $0.025 \mathrm{~nm}$ ) and a Fabry-Pérot optical spectrum analyzer $(120 \mathrm{GHz}$ free spectral range, finesse $\sim 100)$.

The small-signal gain spectrum of our VCSOA was characterized in Ref. 14. The maximum gain was found to be the almost the same for both horizontal and vertical polarizations $(\sim 16.3 \mathrm{~dB})$ thus indicating that dichroism is rather small in our device. On the other side, the gain spectra for the two polarizations were clearly split due to a relatively large birefringence $(\sim 53 \mathrm{GHz})$. These characteristics were also found in the polarization-resolved ASE spectra, which are split in frequency by the same amount and show a slightly higher power $(\approx 7 \%)$ in the horizontal polarization. ${ }^{11-14}$ In both cases, the full width at half maximum (FWHM) of the gain spectra was $\sim 0.7 \mathrm{~nm}$, and the saturation input power was $\sim 1.4 \mathrm{~mW}$ for a beam of diameter $\sim 40 \mu \mathrm{m}$.

In order to characterize XGM in our VCSOA, we first study the efficiency dependence on the injected pump power, for different probe and pump wavelengths, $\lambda_{s}$ and $\lambda_{p}$. The pump beam (diameter $\sim 40 \mu \mathrm{m}$, maximum power of 


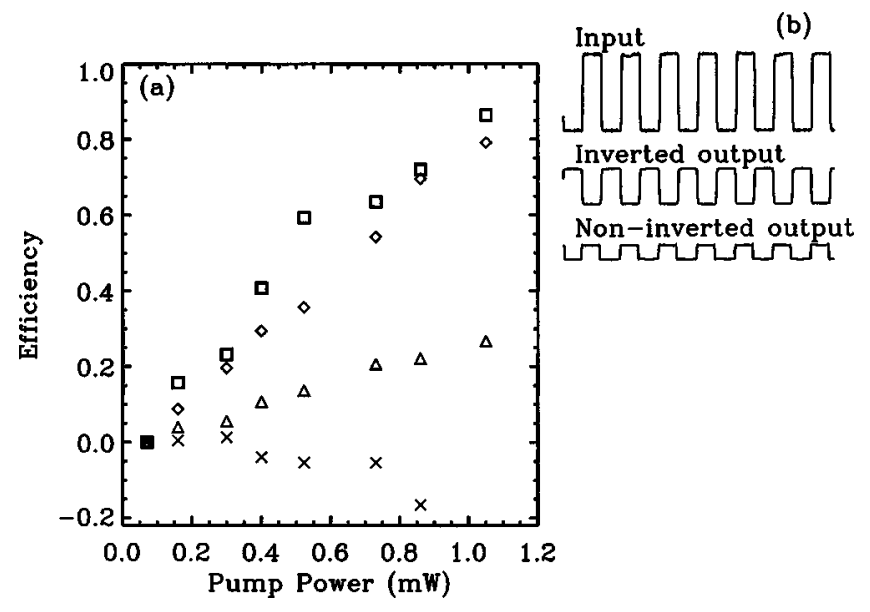

FIG. 1. (a) XGM efficiency as a function of the pump power for different pump $\left(\lambda_{p}\right)$ and probe $\left(\lambda_{s}\right)$ wavelengths: $\lambda_{p}=982.74 \mathrm{~nm}$ and $\lambda_{s}=982.80(\square)$, $982.60(\diamond), 982.50 \mathrm{~nm}(\triangle) ; \lambda_{p}=982.90 \mathrm{~nm}$ and $\lambda_{s}=983.00 \mathrm{~nm}(X)$. The probe power is $0.1 \mathrm{~mW}$, and the peak of the unsaturated gain spectrum occurs at $982.80 \mathrm{~nm}$ for a pump power of $1.5 \mathrm{~mW}$.

$1.5 \mathrm{~mW}$ ) is modulated at $1 \mathrm{kHz}$ by an optical chopper. We define the XGM efficiency as

$$
\eta=1-\frac{P_{\text {probe }}^{\text {on }},}{P_{\text {probe }}^{\text {off }}},
$$

where $P_{\text {probe }}^{\text {on(off) }}$ is the output power at the wavelength of the probe when the pump beam is on (off). $\eta$ thus measures the relative reduction in the power of the amplified probe beam due to the presence of the pump beam. Note, however, that our results effectively correspond to a mixture of XGM and phase modulation, because the VCSOA is operated in reflection mode. The beam reflected from the VCSOA is a superposition of the injected beam and the intracavity field after being reflected and transmitted through the Bragg mirror, respectively. The pump beam modifies the carrier density in the active region, thus both the gain and refractive index of the cavity, hence, the observed modulation is the result of both XGM and phase modulation effects. For the sake of simplicity, we shall nevertheless refer to the observed phenomenon as XGM.

The results for parallel polarizations of the pump and probe beams are shown in Fig. 1. In order to ensure good spatial overlap of the two beams, the diameter of the probe beam is reduced to $\sim 10 \mu \mathrm{m}$. In a first case, we fix $\lambda_{p}$ slightly to the blue of the unsaturated gain peak-which in this case occurs at $\lambda=982.80 \mathrm{~nm}$ - and we scan $\lambda_{s}$ on the blue side of the gain spectrum. In these conditions (see Fig. 1), $\eta$ is positive and proportional to the injected pump power; moreover, it increases as the detuning between the pump and probe beams lowers. The dependence of the modulation efficiency on the wavelength of the probe beam arises from operation of the VCSOA in reflection mode.

An interesting effect occurs when the pump and the probe are detuned slightly to the red of the unsaturated gain peak. In this case, two phenomena are competing; on one side, the aforementioned gain saturation tends to generate an inverted output $(\eta>0)$. On the other side, the redshift of the gain due to the high injected pump power tends to generate a noninverted output $(\eta<0)$ because the amplification of the probe increases. In this case we can see (Fig. 1) that for low pump powers, the gain saturation dominates, and a small Downloaded 10 Jul 2005 to 130.206 .133 .111 . Redistribution subje

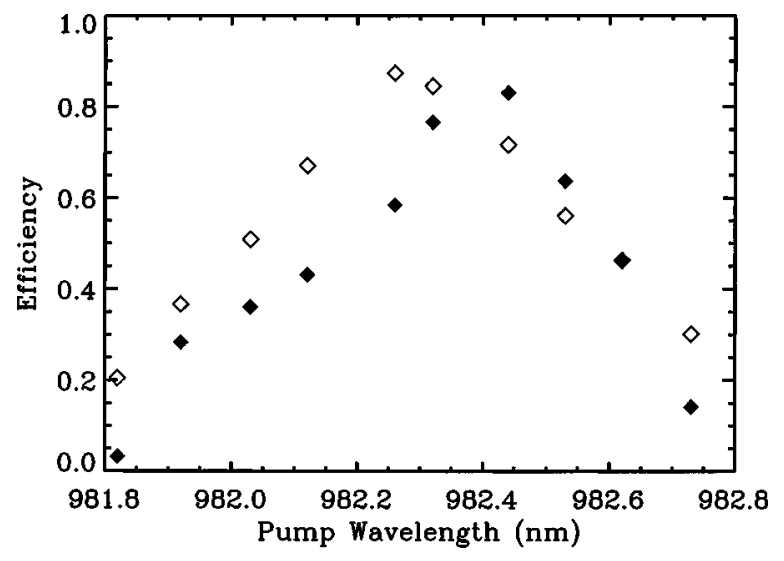

FIG. 2. XGM efficiency vs pump wavelength when the polarization of the probe is parallel/orthogonal to that of the pump (solid/open symbols). The probe wavelength is $982.45 \mathrm{~nm}$ and its power is $0.1 \mathrm{~mW}$.

positive efficiency is observed. However, by further increasing the pump power, the redshift becomes dominant and the efficiency becomes negative. Then, depending on the system parameters we have a wavelength converter showing both negative and positive logic [see Fig. 1(b)].

In a second set of measurements, we characterize the polarization dependence of the efficiency of XGM. We choose the probe wavelength to correspond to the maximum optical gain for its polarization-which in this situation corresponds to $\lambda_{s}=980.45 \mathrm{~nm}$ - and we plot $\eta$ for different wavelengths of the pump beam (see Fig. 2) when its polarization is parallel/orthogonal (solid/open symbols) to that of the probe beam. In the case of parallel polarizations, we can observe that the XGM efficiency is maximum when the wavelength of the pump beam is very close to that of the probe beam, reaching rather high values (up to 90\%) even with our moderate power of the pump beam. As the wavelength detuning between the pump and probe beams increases, the XGM efficiency decreases and it drops to half its maximum value for detuning values of the order of $0.25 \mathrm{~nm}$, thus the FWHM of the XGM efficiency is $\sim 0.5 \mathrm{~nm}$, i.e., over $150 \mathrm{GHz}$. Similar results are obtained when the polarizations of the pump and probe beams are orthogonal (see Fig. 2), although it must be noted that in this case maximum XGM efficiency is not obtained for a zero detuning of the two beams, but when the wavelength of the pump beam corresponds to that of maximum gain for its polarization. This effect is due to the birefringence of the VCSOA cavity ${ }^{10}$ which induces a splitting in the resonance frequency of fields polarized along the two proper polarization axes. Then, when the wavelength of the pump beam corresponds to that of its gain peak, it saturates most efficiently the carrier density in the active region. Interestingly, one can take advantage of this effect to enlarge the optical bandwidth for XGM, which in this cases attains $0.7 \mathrm{~nm}$, i.e., over $215 \mathrm{GHz}$.

In order to estimate the modulation bandwidth for XGM in our device, we substitute the chopper by an electro-optic modulator (EOM) with a rise time of 500 ps to modulate the pump beam. Due to the insertion loss of the EOM, we change the pump laser by a high-power fiber-coupled laser with an in-fiber DBR grating at $\lambda_{p}=980.68 \mathrm{~nm}$. We set the signal wavelength to $981.0 \mathrm{~nm}$, the closest possible with our tunable laser to $\lambda_{p}$, select its polarization to be orthogonal to that of the pump beam, and adjust the VCSOA current to $150 \mathrm{~mA}$ in order to have the signal at the peak of the unsatto AIP license or copyright, see http://apl.aip.org/apl/copyright.jsp 


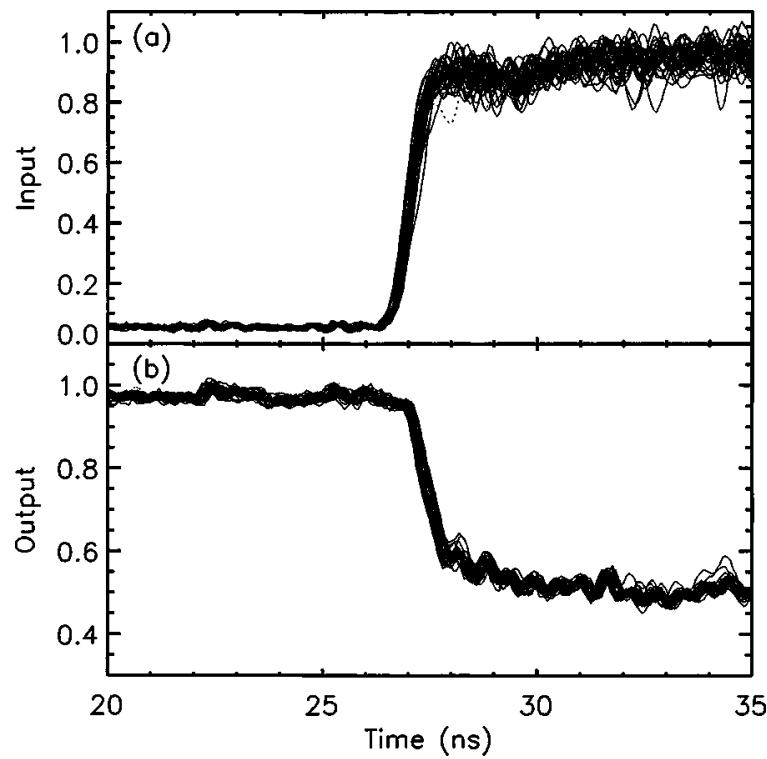

FIG. 3. (a) Modulated pump power and (b) the corresponding response of the probe. The statistics is performed on 2000 events.

urated gain spectrum, where modulation efficiency reaches a maximum. In Fig. 3 we plot the pump modulation and the corresponding output signal. The XGM response time can be estimated by

$$
\tau_{\mathrm{XGM}}=\left(\tau_{s}^{2}-\tau_{p}^{2}\right)^{1 / 2},
$$

where $\tau_{p, s}$ are the rise time of the pump beam and the output beam (i.e., the reflected beam at the signal wavelength), respectively. By performing statistics over 2000 pulses, we measure a mean rise time of the pump and output beams of 575 and $608 \mathrm{ps}$, respectively, which yield a XGM response time of $200 \mathrm{ps}$, thus allowing for $2.5 \mathrm{~Gb} / \mathrm{s}$ modulation, with a jitter lower than $10 \%$.

In conclusion, we have demonstrated that broad-area VCSOAs allow for wavelength conversion via XGM at $2.5 \mathrm{~Gb} / \mathrm{s}$. Modest saturation powers of only $1.5 \mathrm{~mW}$ allow for efficient XGM over an optical bandwidth of $0.7 \mathrm{~nm}$
$(215 \mathrm{GHz})$ in the case of orthogonal polarizations of the pump and probe beams. For parallel beams, the optical bandwidth is reduced to $0.5 \mathrm{~nm}$. Depending on the injected wavelengths we can obtain inverted or noninverted output due to the interplay between gain saturation and redshift of the gain curve. Therefore, the "broad-area concept" can be combined with the optimization of mirrors reflectivities in order to improve the performances of VCSOAs.

The authors acknowledge financial support from MCYT (Spain) through Project No. TIC2002-04255-C04-03. They also thank Ulm University for providing the device in the framework of the project VISTA and the Institut Non Lineaire de Nice for supplying the electro-optical modulator.

${ }^{1}$ T. Durhuus, B. Mikkelsen, C. Joergensen, S. L. Danielsen, and K. E. Stubkjaer, J. Lightwave Technol. 14, 942-954 (1996).

${ }^{2}$ B. Glance, J. M. Wiesenfeld, U. Koren, A. H. Gnauch, H. B. Presby, and A. Jourdan, Proceedings of the CLEO '92, Anaheim, CA, 1992, paper CPD27.

${ }^{3}$ T. Durhuus, C. Joergensen, B. Mikkelsen, R. J. Pedersen, and K. E. Stubkjaer, IEEE Photonics Technol. Lett. 6, 53 (1994).

${ }^{4}$ M. C. Tatham, IEEE Photonics Technol. Lett. 5, 1303 (1993).

${ }^{5}$ S. Bjorlin, J. Piprek, B. Riou, A. Keating, P. Abraham, Y. C. Chiu, and J. E. Bowers, presented at the 25th Optical Fiber Communications Conference, Baltimore, MD, 2000

${ }^{6}$ D. Wiedenmann, B. Moeller, R. Michalzik, and K. J. Ebeling, Electron. Lett. 32, 342 (1996).

${ }^{7}$ C. Tombling, T. Saito, and T. Mukai, IEEE J. Quantum Electron. 30, 2491 (1994).

${ }^{8}$ E. S. Bjorlin, B. Riou, P. Abraham, J. Piprek, Y. J. Chiu, K. A. Black, A. Keating, and J. Bowers, IEEE J. Quantum Electron. 37, 274 (2001).

${ }^{9}$ D. Wiedenmann, C. Jung, M. Grabherr, R. Jager, U. Martin, R. Michalzik, and K. J. Ebeling, in Proceedings of the CLEO 98 Technical Digest 1998, paper CThM5, p. 378.

${ }^{10}$ A. K. Jansen van Doorn, M. P. van Exter, and J. P. Woerdman, Electron. Lett. 30, 1941 (1994).

${ }^{11}$ T. Ackemann and M. Sondermann, Appl. Phys. Lett. 78, 3574 (2001).

${ }^{12}$ P. Debernardi, G. P. Bava, C. Degen, I. Fischer, and W. Elsasser, IEEE J. Quantum Electron. 38, 73 (2002).

${ }^{13}$ J. P. Hermier, A. Bramati, A. Z. Khoury, V. Josse, E. Giacobino, P. Schnitzer, R. Michalzik, and K. J. Ebeling, IEEE J. Quantum Electron. 37, 87 (2001).

${ }^{14}$ F. Marino and S. Balle, Opt. Commun. 231, 325 (2004). 